\title{
Wirtinger integral inequalities for pseudo-integrals and pseudo-additive measure
}

\author{
Jing Guo ${ }^{1 *}$ (D) and Xianjun Zhur, ${ }^{1,2,3}$
}

\section{"Correspondence:}

guo_jing163@163.com

'School of Software Engineering,

Jinling Institute of Technology,

Nanjing 211169, P.R. China

Full list of author information is

available at the end of the article

\section{Springer}

\begin{abstract}
The main purpose of this paper is to show Wirtinger type inequalities for the pseudo-integral. We are concerned with pseudo-integrals based on the following three canonical cases: in the first case, the real semiring with pseudo-operation is generated by a strictly monotone continuous function $g$; in the second case, the pseudo-operations include a pseudo-multiplication and a power arithmetic addition; in the last case, $\oplus$-measures are interval-valued. Examples are given to illustrate these equalities.
\end{abstract}

MSC: Primary 03E72; secondary 28E10; 26E50; 26D15

Keywords: Pseudo-operations; Pseudo-integral; Interval-valued measure; Pseudo-addition; Pseudo-multiplication; Wirtinger inequality

\section{Introduction}

It is well known that if $u \in C^{1}([0, T], \mathbb{R}), u(0)=u(T)$ and $\int_{0}^{T} u(t) d t=0$, then the following inequality holds:

$$
\int_{0}^{T}|u(t)|^{2} d t \leq \frac{T^{2}}{4 \pi^{2}} \int_{0}^{T}\left|u^{\prime}(t)\right|^{2} d t
$$

with equality if and only if $u(t)=A \sin \left(\frac{2 \pi t}{T}\right)+B \cos \left(\frac{2 \pi t}{T}\right)$, where $A$ and $B$ are constants. If $T=2 \pi$, then Inequality (1) is known in the literature as Wirtinger's inequality; see $[1,2]$. The Wirtinger's inequality and its generalizations have wide applications in $p$ Laplacian systems [3, 4], time-delay systems [5-7], Lurie systems [8], stability criteria $[9,10]$, discrete-time systems $[11,12]$ and so on.

Pseudo-analysis is chosen as the research background of this paper, because it presents a contemporary mathematical theory which has been successfully applied in many practical fields. The last decade has shown an increasing research activity on pseudo-analysis [1317]. As a method to promote the classical mathematical analysis, pseudo-analysis extends the concept of traditional operation to pseudo-operation including pseudo-addition and pseudo-multiplication. Later on, the researchers present pseudo-integrals [16] based on

(c) The Author(s) 2021. This article is licensed under a Creative Commons Attribution 4.0 International License, which permits use sharing, adaptation, distribution and reproduction in any medium or format, as long as you give appropriate credit to the original author(s) and the source, provide a link to the Creative Commons licence, and indicate if changes were made. The images or other third party material in this article are included in the article's Creative Commons licence, unless indicated otherwise in a credit line to the material. If material is not included in the article's Creative Commons licence and your intended use is not permitted by statutory regulation or exceeds the permitted use, you will need to obtain permission directly from the copyright holder. To view a copy of this licence, visit http://creativecommons.org/licenses/by/4.0/. 
the important theory of pseudo-analysis, namely pseudo-operations and interval-valued measure. The pseudo-integral is now emerging as one of the hottest mathematical subjects, many scholars have studied its application and promotion on generalizations of integral inequalities [18-22].

Since the Wirtinger inequality is one of the most important inequalities, this paper studies three Wirtinger type integral inequalities in pseudo-analysis environment. In the first case, we consider a Wirtinger type integral inequality for an applied pseudointegration equipped with a monotonic continuous mapping $g$. In the second case, we study a Wirtinger type integral inequality for the pseudo-integration adopting a semiring ([0.1], sup, $\odot)$ to design the theory. In the last case, we show a Wirtinger type integral inequality with respect to interval-valued $\oplus$-measures. Moreover, several examples are provided for validation.

The paper is organized as follows: Sect. 2 contains some of preliminaries. Section 3 provides generalizations of two Wirtinger type inequalities to pseudo-integrals on a $g$ semiring. Section 4 proves a Wirtinger integral inequality for the pseudo-integral of a realvalued function with respect to the pseudo-additive measure. The conclusion is shown in Sect. 5.

\section{Preliminaries}

In this section, we review some basic notions about pseudo-operations and pseudointegrals, the relevant literature includes $[15,23]$.

An interval $[c, d]$ is a closed subset of $[-\infty,+\infty]$. The complete order in $[c, d]$ is expressed as $\preceq$ which can be the usual order of the real line or can be another order. Also, the total order $\preceq$ is closely connected to the choice of pseudo-addition. Namely, for the semirings of the first and the third class, i.e., for the semirings with idempotent pseudo-addition, total order is induced by the following

$$
x \preceq y \quad \text { if and only if } \quad x \oplus y=x .
$$

For the semiring of the second class given by a generator $g$, total order is given by

$$
x \preceq y \text { if and only if } g(x) \leq g(y) .
$$

Let $[c, d]^{+}=\{x \mid x \in[c, d], \mathbf{0} \preceq x\}$. The structure $([c, d], \oplus, \odot)$, endowed with a pseudoaddition $\oplus$ and a pseudo-multiplication $\odot$, is a semiring (see $[16,24]$ ). The equation $\mathbf{0} \odot i=\mathbf{0}$ holds.

We consider the semiring $([c, d], \oplus, \odot)$ in two situations. In the first situation, let $g$ : $[c, d] \rightarrow[0, \infty]$ be a monotone and continuous mapping and let

$$
x \oplus y=g^{-1}(g(x)+g(y)), \quad x \odot y=g^{-1}(g(x) \cdot g(y)) \quad \text { and } \quad x_{\odot}^{(n)}=g^{-1}\left(g^{n}(x)\right) .
$$

The pseudo-integral for a function $p:[a, b] \rightarrow[c, d]$ is expressed as

$$
\int_{[c, d]}^{\oplus} p(t) d t=g^{-1}\left(\int_{c}^{d} g(p(t)) d t\right)
$$


see for details [17]. The second situation is when

$$
x \oplus y=\max (x, y) \quad \text { and } \quad x \odot y=g^{-1}(g(x) g(y)),
$$

the pseudo-integral for a function $p: \mathbb{R} \rightarrow[c, d]$ is given as

$$
\int_{\mathbb{R}}^{\oplus} p \odot d m=\sup (p(x) \odot \psi(x)),
$$

where the function $\psi$ means sup-measure $m$; see [14].

Theorem 1 ([14]) Let $m$ be a sup-measure on $([0, \infty], \mathcal{D}([0, \infty]))$, where $\mathcal{D}([0, \infty])$ is the Borel $\sigma$-algebra on $[0, \infty], m(C)=\operatorname{ess} \sup _{\mu}(\psi(x) \mid x \in A)$, and $\mu:[0, \infty] \rightarrow[0, \infty]$ is a continuous density. Then, for any pseudo-addition $\oplus$ with a generator $g$ there exists a family $m_{\lambda}$ of $\oplus_{\lambda}$-measure on $([0, \infty), \mathcal{D})$, where $\oplus_{\lambda}$ is generated by $g^{\lambda}$ (the function $g$ of the power $\lambda), \lambda \in(0, \infty)$, such that $\lim _{\lambda \rightarrow \infty} m_{\lambda}=m$.

Theorem 2 ([14] $)$ Let $([0, \infty]$, sup, $\odot)$ be a semiring, when $\odot$ is generated with $g$, i.e., we have $x \odot y=g^{-1}(g(x) g(y))$ for every $x, y \in(0, \infty)$. Let $m$ be the same as in Theorem 1 . Then there exists a family $m_{\lambda}$ of $\oplus_{\lambda}$-measures, where $\oplus_{\lambda}$ is generated by $g^{\lambda}, \lambda \in(0, \infty)$ such that, for every continuous function $p:[0, \infty] \rightarrow[0, \infty]$,

$$
\int^{\text {sup }} p \odot d m=\lim _{\lambda \rightarrow \infty} \int^{\oplus_{\lambda}} p \odot d m_{\lambda}=\lim _{n \rightarrow \infty}\left(g^{\lambda}\right)^{-1}\left(\int g^{\lambda}(p(t)) d t\right) .
$$

Pseudo-operations on nonempty subsets $C$ and $D$ of $[c, d]$ adopts the methods similar to the pseudo-addition and the pseudo-multiplication [14]. If $C$ and $D$ stand for two arbitrary nonempty subsets of $[c, d]$ and $\beta \in[c, d]^{+}$, then

$$
\begin{aligned}
& C \oplus D=\{x \oplus y \mid x \in C \text { and } y \in D\}, \\
& C \odot D=\{x \odot y \mid x \in C \text { and } y \in D\}, \\
& \beta \odot C=\{\beta \odot x \mid x \in C\} .
\end{aligned}
$$

The present paper focuses on the interval operation. Let $I$ be the class of all closed subintervals of $[c, d]^{+}$, i.e.,

$$
I=\left\{[w, e] \mid w \leq e \text { and }[w, e] \subseteq[c, d]^{+}\right\} .
$$

It can be shown (see [13]) that for $C, D \in I$, where $C=\left[d_{1}, h_{1}\right]$ and $D=\left[d_{2}, h_{2}\right]$,

$$
C \oplus D=\left[d_{1} \oplus d_{2}, h_{1} \oplus h_{2}\right], \quad C \odot D=\left[d_{1} \odot d_{2}, h_{1} \odot h_{2}\right] .
$$

For $\beta \in[c, d]^{+}$and $D=\left[d_{2}, h_{2}\right] \in I$, we have $\beta \odot D=\left[\beta \odot d_{2}, \beta \odot h_{2}\right]$.

Theorem 3 ([13]) Let $([c, d], \oplus, \odot)$ be a semiring that belongs to one kind basic categories, where $\oplus=\sup$ or $\oplus$ is equivalent to an increasing generator $g$. Let $p: X \rightarrow[c, d]^{+}$be a 
measurable function. An interval-valued set-function $\bar{\mu}_{\mathcal{M}}^{p}$ based on the pseudo-integral of $p$ with respect to interval-valued $\oplus$-measure given by

$$
\bar{\mu}_{\mathcal{M}}^{p}(C)=\int_{C}^{\oplus} p \odot d \bar{\mu}_{\mathcal{M}}=\left[\int_{C}^{\oplus} p \odot d \mu_{d}, \int_{C}^{\oplus} p \odot d \mu_{u}\right]
$$

where $C \subseteq X$, the properties can be summarized as follows:

(i) $\bar{\mu}_{\mathcal{M}}^{p}(\emptyset)=[0,0]$,

(ii) $\bar{\mu}_{\mathcal{M}}^{p}$ is monotone with respect to $\preceq_{s}$,

(iii) $\bar{\mu}_{\mathcal{M}}^{p}$ is additive,

(iv) $\bar{\mu}_{\mathcal{M}}^{p}$ is $\sigma$ - $\oplus$-additive.

In Eq. (4), the set-function $\bar{\mu}_{\mathcal{M}}^{p}$ is an interval-valued $\oplus$-measure.

\section{Wirtinger integral inequality for pseudo-integrals}

In this section, we discuss Wirtinger integral inequality for pseudo-integrals based on the semiring $([0, T], \oplus, \odot)$ and semiring $([0, T]$, sup, $\odot)$ separately.

Let $U(t)=u^{\prime}(t)$, then $u(t)=\int_{0}^{t} U(s) d s$, and Inequality (1) implies that

$$
\int_{0}^{T} U(t)^{2} d t \geq \frac{4 \pi^{2}}{T^{2}} \int_{0}^{T}\left(\int_{0}^{t} U(s) d s\right)^{2} d t
$$

Theorem 4 Suppose that $U:[0, T] \rightarrow[0, T](T \geq 0)$ is a measurable function. If a generator $g:[0, T] \rightarrow[0,+\infty]$ of the pseudo-addition and the pseudo-multiplication is an increasing function and if $\int_{0}^{t} g(U(s)) d s \in C^{1}([0, T], \mathbb{R}), \int_{0}^{T} g(U(t)) d t=0, \int_{0}^{T}\left(\int_{0}^{t} g(U(s)) d s\right) d t=0$. Then for any $\delta$ - $\oplus$-measure

$$
\int_{[0, T]}^{\oplus}(U(t))_{\odot}^{(2)} d t \geq\left(\frac{4 \pi^{2}}{T^{2}}\right) \int_{[0, T]}^{\oplus}\left(\int_{[0, t]}^{\oplus} U(s) d s\right)_{\odot}^{(2)} d t .
$$

Proof By (5), we obtain

$$
\int_{0}^{T}(g(U(t)))^{2} d t \geq \frac{4 \pi^{2}}{T^{2}}\left(\int_{0}^{T}\left(\int_{0}^{t}(g(U(s))) d s\right)^{2} d t\right) .
$$

Since $g^{-1}$ is an increasing function, one has

$$
g^{-1}\left(\int_{0}^{T}(g(U(t)))^{2} d t\right) \geq\left(\frac{4 \pi^{2}}{T^{2}}\right)\left(g^{-1}\left(\int_{0}^{T}\left(\int_{0}^{t}(g(U(s))) d s\right)^{2} d t\right)\right),
$$

which implies that

$$
\begin{aligned}
& \left.g^{-1}\left(\int_{0}^{T}\left(g\left(g^{-1}(g(U(t)))^{2}\right)\right)\right) d t\right) \\
& \quad \geq\left(\frac{4 \pi^{2}}{T^{2}}\right) g^{-1}\left(\int_{0}^{T}\left(g\left(g^{-1}\left(\int_{0}^{t}(g(U(s))) d s\right)^{2}\right)\right) d t\right) .
\end{aligned}
$$

Hence, we have

$$
\int_{[0, T]}^{\oplus}(U(t))_{\odot}^{(2)} d t
$$




$$
\begin{aligned}
& \geq\left(\frac{4 \pi^{2}}{T^{2}}\right) g^{-1}\left(\int_{0}^{T}\left(g\left(g^{-1}\left(\int_{0}^{t}(g(U(s))) d s\right)^{2}\right)\right) d t\right) \\
& =\left(\frac{4 \pi^{2}}{T^{2}}\right) \int_{[0, T]}^{\oplus}\left(g^{-1}\left(\int_{0}^{t}(g(U(s))) d s\right)^{2}\right) d t \\
& =\left(\frac{4 \pi^{2}}{T^{2}}\right) \int_{[0, T]}^{\oplus}\left(g^{-1}\left(g\left(g^{-1}\left(\int_{0}^{t}(g(U(s))) d s\right)\right)\right)^{2}\right) d t \\
& =\left(\frac{4 \pi^{2}}{T^{2}}\right) \int_{[0, T]}^{\oplus}\left(g^{-1}\left(\int_{0}^{t}(g(U(s))) d s\right)\right)_{\odot}^{(2)} d t \\
& =\left(\frac{4 \pi^{2}}{T^{2}}\right) \int_{[0, T]}^{\oplus}\left(\int_{[0, t]}^{\oplus} U(s) d s\right)_{\odot}^{(2)} d t .
\end{aligned}
$$

The proof is therefore complete.

Example 5 Consider that $g(x)=\ln x, U(t)=\mathrm{e}^{\cos t}$, and $T=2 \pi$. The corresponding pseudooperations are $x \oplus y=x y, x \odot y=\mathrm{e}^{\ln x \cdot \ln y}$. By direct calculation, one has

$$
\begin{aligned}
& \int_{[0, t]}^{\oplus} U(s) d s=\mathrm{e}^{\int_{0}^{t} \ln U(t) d t}=\mathrm{e}^{\sin t} \\
& \int_{[0, T]}^{\oplus}\left(\int_{[0, t]}^{\oplus} U(s) d s\right)_{\odot}^{(2)} d t=g^{-1}\left(\int_{0}^{2 \pi}(\sin t)^{2}\right)=\mathrm{e}^{\pi} \\
& \int_{[0, T]}^{\oplus}(U(t))_{\odot}^{(2)} d t=g^{-1}\left(\int_{0}^{2 \pi}(\cos t)^{2}\right)=\mathrm{e}^{\pi} .
\end{aligned}
$$

So Inequality (6) holds.

Example 6 Consider that $g(x)=\tan x, U(t)=\arctan (\cos t)$, and $T=2 \pi$. The corresponding pseudo-operations are $x \oplus y=\arctan (\tan x+\tan y), x \odot y=\arctan (\tan x \cdot \tan y)$. Since

$$
\int_{[0, T]}^{\oplus}\left(\int_{[0, t]}^{\oplus} U(s) d s\right)_{\odot}^{(2)} d t=\arctan \pi=\int_{[0, T]}^{\oplus}(U(t))_{\odot}^{(2)} d t
$$

Inequality (6) holds.

Now, we give an extension of Wirtinger integral inequality with semiring $([0, T]$, sup, $\odot)$.

Theorem 7 Let $\odot$ be represented by an increasing generator $g$ and let $m$ be a complete sup-measure. Then for any functions $U:[0, T] \rightarrow[0, T], T \geq 0$,

$$
\int_{[0, T]}^{\text {sup }} U_{\odot}^{(2)} \odot d m \geq\left(\frac{4 \pi^{2}}{T^{2}}\right) \int_{[0, T]}^{\text {sup }}\left(\left(\int_{[0, t]}^{\text {sup }} U \odot d m\right)_{\odot}^{(2)}\right) \odot d m
$$

Proof According to Theorem 2, one has

$$
\int_{[0, T]}^{\text {sup }} U_{\odot}^{(2)} \odot d m=\lim _{\lambda \rightarrow \infty}\left(\int_{[0, T]}^{\oplus_{\lambda}} U_{\odot}^{(2)} \odot d m_{\lambda}\right)=\lim _{\lambda \rightarrow \infty}\left(g^{\lambda}\right)^{-1}\left(\int_{0}^{T}\left(g^{\lambda}\left((U(t))^{2}\right)\right) d t\right) .
$$


Since $g$ is increasing, $g^{-1}, g^{\lambda},\left(g^{\lambda}\right)^{-1}$ are also increasing. Thus, by Theorem 4 , we have

$$
\begin{aligned}
& \lim _{\lambda \rightarrow \infty}\left(g^{\lambda}\right)^{-1}\left(\int_{0}^{T}\left(g^{\lambda}\left((U(t))^{2}\right)\right) d t\right) \\
& \quad \geq \lim _{\lambda \rightarrow \infty}\left(\frac{4 \pi^{2}}{T^{2}}\right)\left(g^{\lambda}\right)^{-1} \int_{0}^{T}\left(\left(\int_{0}^{t}\left(g^{\lambda}((U(s)) d s)^{2}\right) d t\right.\right. \\
& \quad=\lim _{\lambda \rightarrow \infty}\left(\frac{4 \pi^{2}}{T^{2}}\right)\left(g^{\lambda}\right)^{-1} \int_{0}^{T}\left(g ^ { \lambda } \left(\left(g^{\lambda}\right)^{-1}\left(\left(\int_{0}^{t}\left(g^{\lambda}((U(s)) d s)_{\odot}^{(2)}\right)\right)\right) d t\right.\right. \\
& =\lim _{\lambda \rightarrow \infty}\left(\frac{4 \pi^{2}}{T^{2}}\right) \int_{[0, T]}^{\oplus_{\lambda}}\left(( g ^ { \lambda } ) ^ { - 1 } \left(\left(\int_{0}^{t}\left(g^{\lambda}((U(s)) d s)_{\odot}^{(2)}\right)\right) \odot d m_{\lambda}\right.\right. \\
& \quad=\lim _{\lambda \rightarrow \infty}\left(\frac{4 \pi^{2}}{T^{2}}\right) \int_{[0, T]}^{\oplus_{\lambda}}\left(( g ^ { \lambda } ) ^ { - 1 } \left(\left(g^{\lambda}\left(\left(g^{\lambda}\right)^{-1}\left(\int_{0}^{t}\left(g^{\lambda}((U(s)) d s)\right)\right)^{2}\right)\right) \odot d m_{\lambda}\right.\right. \\
& =\lim _{\lambda \rightarrow \infty}\left(\frac{4 \pi^{2}}{T^{2}}\right) \int_{[0, T]}^{\oplus \lambda}\left(\left(\int_{[0, t]}^{\oplus \lambda} U \odot d m_{\lambda}\right)_{\odot}^{(2)}\right) \odot d m_{\lambda} \\
& =\left(\frac{4 \pi^{2}}{T^{2}}\right) \int_{[0, T]}^{\text {sup }}\left(\left(\int_{[0, t]}^{\text {sup }} U \odot d m\right)_{\odot}^{(2)}\right) \odot d m .
\end{aligned}
$$

This ends the proof.

\section{Inequalities of Wirtinger type for pseudo-integrals with respect to interval-valued $\oplus$-measures}

This section contains the further results of this paper, i.e., Wirtinger type inequalities based on the interval-valued $\oplus$-measure [19].

Theorem 8 Let $([a, b], \oplus, \odot)$ be a g-semiring. If $u \in C^{1}([0, T], \mathbb{R}), \int_{0}^{T} u(t) d t=0, U=u^{\prime}(t)$, and $U:[a, b] \rightarrow[a, b]$ is a measurable function, then

$$
\int_{[0, T]}^{\oplus} U_{\odot}^{(2)} \odot d \bar{\mu}_{\mathcal{M}} \succeq_{S} \frac{4 \pi^{2}}{T^{2}} \int_{[0, T]}^{\oplus}\left(\int_{[0, t]}^{\oplus} U \odot d \bar{\mu}_{\mathcal{M}}\right)_{\odot}^{(2)} \odot d \bar{\mu}_{\mathcal{M}}
$$

Proof By Theorem 3, we have

$$
\int_{[0, T]}^{\oplus} U_{\odot}^{(2)} \odot d \bar{\mu}_{\mathcal{M}}=\left[\int_{[0, T]}^{\oplus} U_{\odot}^{(2)} \odot d \bar{\mu}_{d}, \int_{[0, T]}^{\oplus} U_{\odot}^{(2)} \odot d \bar{\mu}_{h}\right]
$$

On the other hand,

$$
\begin{aligned}
& \frac{4 \pi^{2}}{T^{2}} \int_{[0, T]}^{\oplus}\left(\int_{[0, t]}^{\oplus} U \odot d \bar{\mu}_{\mathcal{M}}\right)_{\odot}^{(2)} \odot d \bar{\mu}_{\mathcal{M}} \\
&=\frac{4 \pi^{2}}{T^{2}} \int_{[0, T]}^{\oplus}\left[\int_{[0, t]}^{\oplus} U \odot d \bar{\mu}_{d}, \int_{[0, t]}^{\oplus} U \odot d \bar{\mu}_{h}\right] \\
& \odot\left[\int_{[0, t]}^{\oplus} U \odot d \bar{\mu}_{d}, \int_{[0, t]}^{\oplus} U \odot d \bar{\mu}_{h}\right] \odot d \bar{\mu}_{\mathcal{M}} \\
&= \frac{4 \pi^{2}}{T^{2}} \int_{[0, T]}^{\oplus}\left[\left(\int_{[0, t]}^{\oplus} U \odot d \bar{\mu}_{d}\right) \odot\left(\int_{[0, t]}^{\oplus} U \odot d \bar{\mu}_{d}\right),\right.
\end{aligned}
$$


Gro and Thu Journal of Inequalities and Applications

(2021) 2021:118

Page 7 of 11

$$
\begin{aligned}
& \left.\left(\int_{[0, t]}^{\oplus} U \odot d \bar{\mu}_{h}\right) \odot\left(\int_{[0, t]}^{\oplus} U \odot d \bar{\mu}_{h}\right)\right] \odot d \bar{\mu}_{\mathcal{M}} \\
= & \frac{4 \pi^{2}}{T^{2}}\left[\int_{[0, T]}^{\oplus}\left(\int_{[0, t]}^{\oplus} U \odot d \bar{\mu}_{d}\right)_{\odot}^{(2)} \odot d \bar{\mu}_{d}, \int_{[0, T]}^{\oplus}\left(\int_{[0, t]}^{\oplus} U \odot d \bar{\mu}_{h}\right)_{\odot}^{(2)} \odot d \bar{\mu}_{h}\right] .
\end{aligned}
$$

Let

$$
\begin{array}{ll}
d_{1}=\int_{[0, T]}^{\oplus} U_{\odot}^{(2)} d \bar{\mu}_{d}, & d_{2}=\int_{[0, T]}^{\oplus}\left(\int_{[0, t]}^{\oplus} U \odot d \bar{\mu}_{d}\right)_{\odot}^{(2)} \odot d \bar{\mu}_{d}, \\
h_{1}=\int_{[0, T]}^{\oplus} U_{\odot}^{(2)} d \bar{\mu}_{h}, & h_{2}=\int_{[0, T]}^{\oplus}\left(\int_{[0, t]}^{\oplus} U \odot d \bar{\mu}_{h}\right)_{\odot}^{(2)} \odot d \bar{\mu}_{h} .
\end{array}
$$

Since the interval $\left[\int_{[0, T]}^{\oplus} U_{\odot}^{(2)} \odot d \bar{\mu}_{d}, \int_{[0, T]}^{\oplus} U_{\odot}^{(2)} \odot d \bar{\mu}_{h}\right]$ is pseudo-convex, an arbitrary lemont

$$
x \in \int_{[0, T]}^{\oplus} U_{\odot}^{(2)} \odot d \bar{\mu}_{\mathcal{M}}
$$

can be written in the form

$$
x=\alpha \odot \int_{[0, T]}^{\oplus} U_{\odot}^{(2)} \odot d \bar{\mu}_{d} \oplus \beta \odot \int_{[0, T]}^{\oplus} U_{\odot}^{(2)} d \bar{\mu}_{h},
$$

where $\alpha, \beta \in[a, b]^{+}, \alpha \oplus \beta=1$.

Based on Wirtinger inequality for pseudo-integrals (6), one has

$$
\int_{[0, T]}^{\oplus} U_{\odot}^{(2)} \odot d \bar{\mu}_{d} \succeq \frac{4 \pi^{2}}{T^{2}} \int_{[0, T]}^{\oplus}\left(\int_{[0, t]}^{\oplus} U \odot d \bar{\mu}_{d}\right)_{\odot}^{(2)} \odot d \bar{\mu}_{d} .
$$

Since $\alpha \in[a, b]^{+}$and $\odot$ is a positively non-decreasing function, we have

$$
\alpha \odot \int_{[0, T]}^{\oplus} U_{\odot}^{(2)} \odot d \bar{\mu}_{d} \succeq \alpha \odot \frac{4 \pi^{2}}{T^{2}} \int_{[0, T]}^{\oplus}\left(\int_{[0, t]}^{\oplus} U \odot d \bar{\mu}_{d}\right)_{\odot}^{(2)} \odot d \bar{\mu}_{d} .
$$

Similarly, for $\beta \in[a, b]^{+}$and $\odot$-measure $\mu_{h}, \odot$ is a positively non-decreasing function, and then, by Inequality (6), we have

$$
\beta \odot \int_{[0, T]}^{\oplus} U_{\odot}^{(2)} \odot d \bar{\mu}_{h} \succeq \beta \odot \frac{4 \pi^{2}}{T^{2}} \int_{[0, T]}^{\oplus}\left(\int_{[0, t]}^{\oplus} U \odot d \bar{\mu}_{h}\right)_{\odot}^{(2)} \odot d \bar{\mu}_{h} .
$$

For

$$
\begin{aligned}
y= & \alpha \odot \frac{4 \pi^{2}}{T^{2}} \int_{[0, T]}^{\oplus}\left(\int_{[0, t]}^{\oplus} U \odot d \bar{\mu}_{d}\right)_{\odot}^{(2)} \odot d \bar{\mu}_{d} \\
& \oplus \beta \odot \frac{4 \pi^{2}}{T^{2}} \int_{[0, T]}^{\oplus}\left(\int_{[0, t]}^{\oplus} U \odot d \bar{\mu}_{h}\right)_{\odot}^{(2)} \odot d \bar{\mu}_{h},
\end{aligned}
$$

we have $x \succeq y$ and

$$
y \in \frac{4 \pi^{2}}{T^{2}} \int_{[0, T]}^{\oplus}\left(\int_{[0, t]}^{\oplus} U \odot d \bar{\mu}_{\mathcal{M}}\right)_{\odot}^{(2)} \odot d \bar{\mu}_{\mathcal{M}} .
$$


Therefore, we have completed the first part of the proof. Furthermore, due to the property of the pseudo-convexity of the subinterval of $[a, b]^{+}$,

$$
y \in \frac{4 \pi^{2}}{T^{2}} \int_{[0, T]}^{\oplus}\left(\int_{[0, t]}^{\oplus} U \odot d \bar{\mu}_{\mathcal{M}}\right)_{\odot}^{(2)} \odot d \bar{\mu}_{\mathcal{M}}
$$

can be written in the form

$$
\begin{aligned}
y= & \alpha \odot \frac{4 \pi^{2}}{T^{2}} \int_{[0, T]}^{\oplus}\left(\int_{[0, t]}^{\oplus} U \odot d \bar{\mu}_{d}\right)_{\odot}^{(2)} \odot d \bar{\mu}_{d} \\
& \oplus \beta \odot \frac{4 \pi^{2}}{T^{2}} \int_{[0, T]}^{\oplus}\left(\int_{[0, t]}^{\oplus} U \odot d \bar{\mu}_{h}\right)_{\odot}^{(2)} \odot d \bar{\mu}_{h},
\end{aligned}
$$

for some $\alpha, \beta \in[a, b]^{+}, \alpha \oplus \beta=1$. It follows from (6) that

$$
\begin{aligned}
& \int_{[0, T]}^{\oplus} U_{\odot}^{(2)} \odot d \bar{\mu}_{d} \succeq \frac{4 \pi^{2}}{T^{2}} \int_{[0, T]}^{\oplus}\left(\int_{[0, t]}^{\oplus} U \odot d \bar{\mu}_{d}\right)_{\odot}^{(2)} \odot d \bar{\mu}_{d}, \\
& \int_{[0, T]}^{\oplus} U_{\odot}^{(2)} \odot d \bar{\mu}_{h} \succeq \frac{4 \pi^{2}}{T^{2}} \int_{[0, T]}^{\oplus}\left(\int_{[0, t]}^{\oplus} U \odot d \bar{\mu}_{h}\right)_{\odot}^{(2)} \odot d \bar{\mu}_{h} .
\end{aligned}
$$

Since $\oplus$ is a non-decreasing function, and $\odot$ is a positively non-decreasing function, one has

$$
\begin{gathered}
\alpha \odot \int_{[0, T]}^{\oplus} U_{\odot}^{(2)} \odot d \bar{\mu}_{d} \oplus \beta \odot \int_{[0, T]}^{\oplus} U_{\odot}^{(2)} d \bar{\mu}_{h} \\
\succeq \alpha \odot \frac{4 \pi^{2}}{T^{2}} \int_{[0, T]}^{\oplus}\left(\int_{[0, t]}^{\oplus} U \odot d \bar{\mu}_{d}\right)_{\odot}^{(2)} \odot d \bar{\mu}_{d} \\
\oplus \beta \odot \frac{4 \pi^{2}}{T^{2}} \int_{[0, T]}^{\oplus}\left(\int_{[0, t]}^{\oplus} U \odot d \bar{\mu}_{h}\right)_{\odot}^{(2)} \odot d \bar{\mu}_{h} .
\end{gathered}
$$

This yields

$$
x=\alpha \odot \int_{[0, T]}^{\oplus} U_{\odot}^{(2)} \odot d \bar{\mu}_{d} \oplus \beta \odot \int_{[0, T]}^{\oplus} U_{\odot}^{(2)} d \bar{\mu}_{h} \in \int_{[0, T]}^{\oplus} U_{\odot}^{(2)} d \bar{\mu}_{\mathcal{M}}
$$

satisfying $x \succeq y$. Hence, the following form holds:

$$
\left[d_{1}, h_{1}\right] \succeq_{S}\left[d_{2}, h_{2}\right] .
$$

This completes the proof.

Remark 9 The proof for a decreasing generator $g$ is similar. In this case, since the total order is opposite to the usual order on the real line, Inequality (7) is reduced to

$$
\left[d_{1}, h_{1}\right] \preceq_{S}\left[d_{2}, h_{2}\right] .
$$


Example 10 Consider the $g$-semiring with the generating function $g(x)=x^{2}$, the intervalvalued $\oplus$-measure $\bar{\mu}((a, b])=\left[\sqrt{\frac{2}{3}(b-a)}, \sqrt{b-a}\right],(a, b] \in B[a, b]$, the function $u(x)=\frac{1}{2}$, if $0 \leq x \leq \frac{1}{2}, u(x)=1$, if $\frac{1}{2} \leq x \leq 1$ and $T=1$. Then, the Wirtinger inequality (7) is of the form

$$
\int_{[0,1]}^{\oplus} U_{\odot}^{(2)} \odot d \bar{\mu}_{\mathcal{M}} \geq_{S} 4 \pi^{2} \int_{[0,1]}^{\oplus}\left(\int_{[0, t]}^{\oplus} U \odot d \bar{\mu}_{\mathcal{M}}\right)_{\odot}^{(2)} \odot d \bar{\mu}_{\mathcal{M}}
$$

The pseudo-integral of the function $U$ with respect to the interval-valued $\oplus$-measure $\bar{\mu}_{\mathcal{M}}$ is

$$
\int_{[0,1]}^{\oplus} U \odot d \bar{\mu}_{\mathcal{M}}=\left[\int_{[0,1]}^{\oplus} U \odot d \bar{\mu}_{d}, \int_{[0,1]}^{\oplus} U \odot d \bar{\mu}_{h}\right]
$$

Since $g(x)=x^{2}, x \odot y=x \cdot y, x \oplus y=\sqrt[\alpha]{x^{\alpha}+y^{\alpha}}$, one has

$$
\begin{aligned}
\int_{[0,1]}^{\oplus} U \odot d \bar{\mu}_{d} & =\frac{1}{2} \odot \bar{\mu}_{d}\left(\left[0, \frac{1}{2}\right]\right) \oplus 1 \odot \bar{\mu}_{d}\left(\left(\frac{1}{2}, 1\right]\right) \\
& =\frac{1}{2} \odot \sqrt{\frac{2}{3} \cdot \frac{1}{2}} \oplus 1 \odot \sqrt{\frac{2}{3} \cdot \frac{1}{2}}=\sqrt{\frac{5}{12}}, \\
\int_{[0,1]}^{\oplus} U \odot d \bar{\mu}_{h} & =\frac{1}{2} \odot \bar{\mu}_{h}\left(\left[0, \frac{1}{2}\right]\right) \oplus 1 \odot \bar{\mu}_{h}\left(\left(\frac{1}{2}, 1\right]\right) \\
& =\frac{1}{2} \odot \sqrt{\frac{1}{2}} \oplus 1 \odot \sqrt{\frac{1}{2}}=\sqrt{\frac{5}{8}} .
\end{aligned}
$$

It follows that

$$
\begin{aligned}
& \int_{[0,1]}^{\oplus} U \odot d \bar{\mu}_{\mathcal{M}}=\left[\sqrt{\frac{5}{12}}, \sqrt{\frac{5}{8}}\right], \\
& \left(\int_{[0,1]}^{\oplus} U \odot d \bar{\mu}_{\mathcal{M}}\right)_{\odot}^{(2)}=\left[\frac{5}{12}, \frac{5}{8}\right], \\
& \int_{[0,1]}^{\oplus}\left(\int_{[0, t]}^{\oplus} U \odot d \bar{\mu}_{d}\right)_{\odot}^{(2)} \odot d \bar{\mu}_{d}=\frac{5}{12} \odot \bar{\mu}_{d}[0,1]=\frac{5}{12} \sqrt{\frac{2}{3}}, \\
& \int_{[0, T]}^{\oplus}\left(\int_{[0, t]}^{\oplus} U \odot d \bar{\mu}_{h}\right)_{\odot}^{(2)} \odot d \bar{\mu}_{h}=\frac{5}{8} \odot \bar{\mu}_{h}[0,1]=\frac{5}{8} .
\end{aligned}
$$

For the right hand side of (8), we have

$$
\begin{aligned}
4 & \pi^{2} \int_{[0,1]}^{\oplus}\left(\int_{[0, t]}^{\oplus} U \odot d \bar{\mu}_{\mathcal{M}}\right)_{\odot}^{(2)} \odot d \bar{\mu}_{\mathcal{M}} \\
& =\frac{4 \pi^{2}}{T^{2}}\left[\int_{[0, T]}^{\oplus}\left(\int_{[0, t]}^{\oplus} U \odot d \bar{\mu}_{d}\right)_{\odot}^{(2)} \odot d \bar{\mu}_{d}, \int_{[0, T]}^{\oplus}\left(\int_{[0, t]}^{\oplus} U \odot d \bar{\mu}_{h}\right)_{\odot}^{(2)} \odot d \bar{\mu}_{h}\right] \\
& =\left[\frac{3}{5} \sqrt{\left.\frac{2}{3} \pi, \frac{5}{2} \pi\right] .}\right.
\end{aligned}
$$


Since $u(x)=\frac{1}{4}, 0 \leq x \leq \frac{1}{2}, u(x)=1, \frac{1}{2} \leq x \leq 1$, the following equations hold:

$$
\begin{aligned}
\int_{[0,1]}^{\oplus} U_{\odot}^{(2)} \odot d \bar{\mu}_{d} & =\frac{1}{4} \odot \bar{\mu}_{d}\left(\left[0, \frac{1}{2}\right]\right) \oplus 1 \odot \bar{\mu}_{d}\left(\left(\frac{1}{2}, 1\right]\right) \\
& =\frac{1}{4} \odot \sqrt{\frac{2}{3} \cdot \frac{1}{2}} \oplus 1 \odot \sqrt{\frac{2}{3} \cdot \frac{1}{2}}=\frac{1}{4} \sqrt{\frac{17}{3}} \\
\int_{[0,1]}^{\oplus} U_{\odot}^{(2)} \odot d \bar{\mu}_{h} & =\frac{1}{4} \odot \bar{\mu}_{h}\left(\left[0, \frac{1}{2}\right]\right) \oplus 1 \odot \bar{\mu}_{h}\left(\left(\frac{1}{2}, 1\right]\right) \\
& =\frac{1}{4} \odot \sqrt{\frac{1}{2}} \oplus 1 \odot \sqrt{\frac{1}{2}}=\frac{1}{4} \sqrt{\frac{17}{2}} .
\end{aligned}
$$

For the left hand of (8), we have

$$
\int_{[0,1]}^{\oplus} U_{\odot}^{(2)} \odot d \bar{\mu}_{\mathcal{M}}=\left[\int_{[0,1]}^{\oplus} U_{\odot}^{(2)} \odot d \bar{\mu}_{d}, \int_{[0,1]}^{\oplus} U_{\odot}^{(2)} \odot d \bar{\mu}_{h}\right]=\left[\frac{1}{4} \sqrt{\frac{17}{3}}, \frac{1}{4} \sqrt{\frac{17}{2}}\right] .
$$

The final form of $(8)$ is

$$
\left[\frac{3}{5} \sqrt{\frac{2}{3}} \pi, \frac{5}{2} \pi\right] \succeq_{S}\left[\frac{1}{4} \sqrt{\frac{17}{3}}, \frac{1}{4} \sqrt{\frac{17}{2}}\right]
$$

\section{Conclusion}

In this paper, we present three Wirtinger type integral inequalities for pseudo-integrals with $g$-semirings and interval-valued $\oplus$-measure, respectively. It is well known that pseudo-integrals cover Lebesgue integrals, Sugeno fuzzy integrals, so the presented process can extended to other integrals. Since the Wirtinger integral inequalities are important basic tools widely used in engineering technology, and that the pseudo-integral is applicable in many fields by helping to explain some practical problems, our inequalities could become essential tools in several practical areas, such as stability analysis of delayed stochastic neural networks and the Takagi-Sugeno fuzzy time-delay system. Thus, in future work, we intend to refine our proposed inequalities and to modify the Wirtinger type double integral inequality for pseudo-integrals, and then apply them to fuzzy time-varying delay system.

\section{Acknowledgements}

The authors would like to thank all the benefactors for their remarkable comments, suggestions, and ideas, which helped improve this paper.

\section{Funding}

This research was financially supported by Universities' Philosophy and Social Science Researches in Jiangsu Province (No.: 2020SJA0534) and Research Initiation Fund for High-level Talents of Jinling Institute Technology (No.: jit-b-201817), China Postdoctoral Science Foundation (No.: 2020T130129ZX).

\section{Abbreviations}

Not applicable.

Availability of data and materials

Not applicable.

Ethics approval and consent to participate

Not applicable. 


\section{Consent for publication}

Not applicable.

\section{Authors' contributions}

The authors read and approved the final manuscript. All authors contributed equally to the writing of this paper.

\section{Author details}

${ }^{1}$ School of Software Engineering, Jinling Institute of Technology, Nanjing 211169, P.R. China. ${ }^{2}$ Reproductive Medicine Center, Jinling Hospital, Nanjing 210002, P.R. China. ${ }^{3}$ Research Center for New Technology in Intelligent Equipment, Nanjing University, Nanjing 210093, P.R. China.

\section{Publisher's Note}

Springer Nature remains neutral with regard to jurisdictional claims in published maps and institutional affiliations.

Received: 25 January 2021 Accepted: 23 June 2021 Published online: 13 July 2021

\section{References}

1. Hardy, G.H., Littlewood, J.E., Pólya, G.: Inequalities, 2nd edn. Cambridge University Press, Cambridge (1952)

2. Beckenbach, E.F., Bellman, R.: Inequalities, Second revised printing. Springer, New York (1965)

3. Yoshida, N.: A Picone identity for half-linear elliptic equations and its applications to oscillation theory. Nonlinear Anal. 71(10), 4935-4951 (2009). https://doi.org/10.1016/j.na.2009.03.051

4. Teng, K.-M., Wu, X.: Existence and subharmonicity of solutions for nonlinear non-smooth periodic systems with a p-Laplacian. Nonlinear Anal. 68(12), 3742-3756 (2008). https://doi.org/10.1016/j.na.2007.04.016

5. Gyurkovics, E.: A note on Wirtinger-type integral inequalities for time-delay systems. Automatica 61, 44-46 (2015). https://doi.org/10.1016/j.automatica.2015.07.033

6. Ding, S., Wang, Z., Zhang, H.: Wirtinger-based multiple integral inequality for stability of time-delay systems. Int. J. Control 91(1), 12-18 (2018). https://doi.org/10.1080/00207179.2016.1266516

7. Seuret, A., Gouaisbaut, F.: Wirtinger-based integral inequality: application to time-delay systems. Automatica 49(9), 2860-2866 (2013). https://doi.org/10.1016/j.automatica.2013.05.030

8. Zhang, L., Wang, S., Yu, W., Song, Y.: New absolute stability results for Lurie systems with interval time-varying delay based on improved Wirtinger-type integral inequality. Int. J. Robust Nonlinear Control 29(8), 2422-2437 (2019). https://doi.org/10.1002/rnc.4526

9. Lee, T.H., Park, J.H., Park, M.-J., Kwon, O.-M., Jung, H.-Y.: On stability criteria for neural networks with time-varying delay using Wirtinger-based multiple integral inequality. J. Franklin Inst. 352(12), 5627-5645 (2015). https://doi.org/10.1016/j.jfranklin.2015.08.024

10. Kwon, O.M., Park, M.J., Park, J.H., Lee, S.M., Cha, E.J.: Improved results on stability of linear systems with time-varying delays via Wirtinger-based integral inequality. J. Franklin Inst. 351(12), 5386-5398 (2014). https://doi.org/10.1016/j.jranklin.2014.09.021

11. Ding, S., Wang, Z., Xie, X.: Periodic event-triggered synchronization for discrete-time complex dynamical networks. IEEE Transactions on Neural Networks and Learning Systems, 1-12 (2021). https://doi.org/10.1109/TNNLS.2021.3053652

12. Ding, S., Xie, X., Liu, Y.: Event-triggered static/dynamic feedback control for discrete-time linear systems. Inf. Sci. 524 33-45 (2020). https://doi.org/10.1016/j.ins.2020.03.044

13. Grbić, T., Štajner-Papuga, I., Štrboja, M.: An approach to pseudo-integration of set-valued functions. Inf. Sci. 181(11), 2278-2292 (2011). https://doi.org/10.1016/j.ins.2011.01.038

14. Mesiar, R., Pap, E.: Idempotent integral as limit of $g$-integrals. Fuzzy Sets Syst. 102(3), 385-392 (1999). https://doi.org/10.1016/S0165-0114(98)00213-9

15. Pap, E.: Generalized real analysis and its applications. Int. J. Approx. Reason. 47(3), 368-386 (2008). https://doi.org/10.1016/j.ijar.2007.05.015

16. Pap, E.: $\sigma$-null-additive set functions. Novi Sad J. Math. 32(1), 47-57 (2002)

17. Pap, E.: An integral generated by a decomposable measure. Zb. Rad. Prir.-Mat. Fak., Ser. Mat. 20(1), 135-144 (1990)

18. Lu, W., Song, X., Yang, X.: Inequalities of Barnes-Godunova-Levin and Lyapunov type for interval-valued measures based on pseudo-integrals. Nanjing Daxue Xuebao Shuxue Bannian Kan 33(1), 40-56 (2016)

19. Medić, S., Grbić, T., Perović, A., Nikoličić, S.: Inequalities of Hölder and Minkowski type for pseudo-integrals with respect to interval-valued $\oplus$-measures. Fuzzy Sets Syst. 304, 110-130 (2016). https://doi.org/10.1016/j.fss.2015.11.014

20. Štrboja, M., Grbić, T., Štajner-Papuga, I., Grujić, G., Medić, S.: Jensen and Chebyshev inequalities for pseudo-integrals of set-valued functions. Fuzzy Sets Syst. 222, 18-32 (2013). https://doi.org/10.1016/j.fss.2012.07.011

21. Agahi, H., Ouyang, Y., Mesiar, R., Pap, E., Strboja, M.: Hölder and Minkowski type inequalities for pseudo-integral. Appl. Math. Comput. 217(21), 8630-8639 (2011). https://doi.org/10.1016/j.amc.2011.03.100

22. Agahi, H., Mesiar, R., Ouyang, Y.: Chebyshev type inequalities for pseudo-integrals. Nonlinear Anal. 72(6), 2737-2743 (2010). https://doi.org/10.1016/j.na.2009.11.017

23. Sugeno, M., Murofushi, T.: Pseudo-additive measures and integrals. J. Math. Anal. Appl. 122(1), 197-222 (1987). https://doi.org/10.1016/0022-247X(87)90354-4

24. Kuich, W., Salomaa, A.: Semirings, Automata, Languages. Springer, Berlin (1986). https://doi.org/10.1007/978-3-642-69959-7 